\title{
Pequenas espécies de Parmeliaceae (Ascomycota) eciliadas no Parque Estadual da Cantareira, estado de São Paulo, Brasil: gêneros Canoparmelia e Crespoa
}

Small eciliate Parmeliaceae species (Ascomycota) in Parque Estadual da Cantareira, São Paulo State, Brazil: the genera Canoparmelia and Crespoa

Michel Navarro Benatti ${ }^{1,2}$

\begin{abstract}
Resumo
O levantamento das espécies pertencentes aos gêneros Canoparmelia e Crespoa no Parque Estadual da Cantareira e arredores revelou a ocorrência, respectivamente, de quatro e três espécies. As espécies são reconhecidas pelos talos foliosos com lobos arredondados ou \pm truncados estreitos em geral $\leq 0,5 \mathrm{~cm}$ larg., diferindo pela morfologia e anatomia dos córtices superiores e dos metabólitos secundários, sendo que nenhuma espécie dos dois gêneros tende a formar cílios marginais. Constituintes químicos medulares são diversos. São apresentados uma chave de identificação, descrições, comentários e ilustrações para as espécies citadas. Palavras-chave: Serra da Cantareira, margens eciliadas, fungos liquenizados.
\end{abstract}

\begin{abstract}
The survey of the species belonging to the genera Canoparmelia and Crespoa in the Parque Estadual da Cantareira and adjacencies revealed the respective occurrence of four and three species. The species are recognized by foliose thalli with narrow rounded or \pm truncate lobes generally $\leq 0.5 \mathrm{~cm}$ wide, differing by the upper cortex morphology and anatomy as well as by the secondary metabolites, all species of the genus having eciliate margins. Medullary chemical constituents are diverse. A key for identification, descriptions, comments and illustrations are provided for the cited species.
\end{abstract}

Key words: Cantareira Mountain Range, eciliate margins, lichenized fungi.

\section{Introdução}

O gênero Canoparmelia Elix et al. (1986) caracteriza-se por apresentar espécies relacionadas a Parmelia texana e lobos pequenos e arredondados, atranorina cortical, ausência da formação de cílios marginais, córtex inferior negro com rizinas simples com uma estreita margem inferior nua e conídios fusiformes a bifusiformes. Atualmente, são conhecidas cerca de 40 espécies mundialmente, das quais cinco são citadas para o Brasil, excluindo-se aqui três espécies citadas por Marcelli (2004), recentemente combinadas em Crespoa (D. Hawksw.) Lendemer \& Hodkinson.

O gênero Crespoa foi descrito recentemente (Lendemer \& Hodkinson 2012) a partir do grupo de espécies de Crespoa crozalsiana, morfologicamente semelhantes a
Canoparmelia, do qual foi segregado, porém de talo caracteristicamente escrobiculado, sendo composto no momento por cinco espécies, das quais três ocorrem no Brasil (Marcelli 2004; Lendemer \& Hodkinson 2012; Benatti \& Lendemer 2014).

Dados aproximados do número de espécies de fungos liquenizados para o Brasil indicam para a Mata Atlântica um total de 400 espécies publicadas em uma estimativa total de 850 espécies esperadas para a região na qual está inclusa a Serra da Cantareira (Marcelli 1998a). Previamente aos estudos florísticos que estão sendo feitos para a região da Serra da Cantareira, conhecia-se apenas uma relação de 111 espécies de fungos liquenizados pertencentes a 47 gêneros (Marcelli 1998b), sem maiores detalhes sobre as espécies. Dando continuidade aos estudos com os

\footnotetext{
'Instituto de Botânica, Núcleo de Pesquisa em Micologia, C.P. 3005, 01061-970, São Paulo, SP, Brasil

${ }^{2}$ Autor para correspondência: michel_benatti@yahoo.com.br
} 
liquens do Parque Estadual da Cantareira, já foram publicados um artigo com as pequenas espécies de Parmeliaceae ciliadas da mesma localidade (Benatti 2012), outro abordando as espécies de cianoliquens (Benatti et al. 2013a), outros que abordam as espécies de Parmotrema (Benatti 2014a,b) e um com espécies de três gêneros de Physciaceae (Benatti \& Jungbluth 2014) que incluem 42 espécies pertencentes a dez gêneros, somando ao todo 25 espécies à lista.

Este trabalho objetivou realizar o levantamento das espécies foliosas de Canoparmelia Elix \& Hale e Crespoa (D. hawksw.) Lendemer \& Hodkinson que ocorrem no Parque Estadual da Cantareira e regiões circunvizinhas. São fornecidos uma chave artificial de identificação, descrições comentadas, dados das localidades e de ambientes para as espécies encontradas.

\section{Material e Métodos}

A metodologia de coleta para fungos liquenizados foliosos é descrita em Hale (1987), Malcolm \& Galloway (1997) e Benatti \& Marcelli (2007). As análises morfológicas consistiram na observação das características macro e microscópicas dos espécimes, através de estereomicroscópio e microscópio óptico. Foram analisadas as estruturas somáticas e de reprodução, tanto direta como indireta dos talos.

O material coletado na área da Reserva da Serra da Cantareira e depositado no herbário Maria Eneyda P. Kauffmann Fidalgo (SP) do Instituto de Botânica foi identificado conforme a metodologia tradicional de liquenologia, descrita em Fink (1905), Hale (1979) e Galloway $(1985,2007)$. Os dados históricos, geográficos e climatológicos a respeito da localidade de estudo podem ser visualizados em Benatti (2012)

A metodologia de análises químicas consistiu em testes de coloração, irradiação por luz UV, testes de microcristalização com reagentes G.E. e G.A.W., e cromatografia em camada delgada (CCD) com solvente C, seguindo Asahina \& Shibata (1954), Walker \& James (1980), White \& James (1985), Huneck \& Yoshimura (1996), Bungartz (2001) e Orange et al. (2001).

\section{Resultados e Discussão}

Foi analisado um total de 60 amostras de fungos liquenizados foliosos pertencentes aos gêneros Canoparmelia e Crespoa da família Parmeliaceae. No Parque Estadual da Cantareira, foram encontradas sete espécies, sendo quatro de Canoparmelia e três de Crespoa. Embora todas sejam conhecidas para o estado, existem poucas citações (vide distribuições geográficas das espécies) e este é o primeiro registro para a localidade de estudo, uma densa área florestal em meio à maior região urbana da América do Sul, e o primeiro registro em uma reserva de Mata Atlântica em meio urbano no Brasil. Uma das espécies encontradas foi recentemente descrita para a área de estudo, sua localidade tipo (Benatti et al. 2008).

Canoparmelia amazonica (Nyl.) Elix \& Hale, C. caroliniana (Nyl.) Elix \& Hale e C. sanguinea Marcelli Benatti \& Elix apresentam formação de isídios, enquanto Crespoa carneopruinata (Zahlbr.) Lendemer \& Hodkinson, C. crozalsiana (de Lesd.) Lendemer \& Hodkinson e Canoparmelia texana (Tuck.) Elix \& Hale apresentam formação de sorédios. Somente uma espécie que forma apenas apotécios foi encontrada, Crespoa scrobicularis (Kremp.) Benatti \& Lendemer. Todos os espécimes encontrados são corticícolas em troncos, galhos ou ramos de árvores, sendo que alguns espécimes de $C$. crozalsiana também foram encontrados sobre rocha.

Crespoa carneopruinata, C. crozalsiana e C. scrobicularis apresentam ácidos do complexo estíctico e ácido menegaziaico medulares. As espécies de Canoparmelia apresentam química mais diversa, incluindo ácido perlatólico $(C$. caroliniana), ácido divaricático (C. texana), ácido protocetrárico $(C$. amazonica) e, no caso de $C$. sanguinea, um complexo contendo diversos ácidos, sendo o principal o ácido olivetolcarboxílico (Benatti et al. 2008).

Poucos espécimes apresentaram formação de apotécios e somente em alguns foram visualizados ascósporos maduros. Embora alguns espécimes tenham apresentado picnídios, não foram encontrados conídios em vários dos materiais. A exceção foi no caso de alguns espécimes de $C$. caroliniana e de C. scrobicularis, nos quais foram encontrados ascósporos e conídios. 


\section{Chave de identificação para as espécies de Canoparmelia e Crespoa do Parque Estadual da Cantareira}

1. Lobos foveolados ou escrobiculados 2

2. Talo sem sorédios ou isídios, normalmente com apotécios Crespoa scrobicularis

2'. Talo sorediado, às vezes com apotécios 3

3. Talo laciniado, lacínias 0,5-2 mm larg. e de ápices truncados, sorais com frequência acompanhando as quebras do córtex Crespoa carneopruinata

3'. Talo lobado, lobos 2-5 mm larg. e de ápices arredondados, sorais capitados as vezes aglomerados irregularmente Crespoa crozalsiana

1'. Lobos lisos a rugosos 4

4. Talo sorediado (medula C-, KC- ou + violáceo, ácido divaricático) Canoparmelia texana

4'. Talo isidiado (medula com outros ácidos)

5. Medula $\mathrm{K}+$ amarelado, $\mathrm{KC}+$ róseo, $\mathrm{P}+$ alaranjado (ácido protocetrárico)

Canoparmelia amazonica

5'. Medula K-, C+ e/ou KC+ 6

6. Medula $\mathrm{C}+$ vermelho, $\mathrm{KC}+$ vermelho (complexo formado por diversos ácidos)

Canoparmelia sanguinea

6'. Medula C-, $\mathrm{KC}+$ violáceo (ácido perlatólico)

Canoparmelia caroliniana

Canoparmelia amazonica (Nyl.) Elix \& Hale, Mycotaxon 27: 278. 1986. $\equiv$ Parmelia amazonica Nyl., Flora 68: 611. 1885. Tipo: BRASIL. PARÁ: Santarém, leg. Spruce 111 (H-Nyl 35111, lectótipo n.v., BM, G, NY, PC, W, duplicatas n.v.).

Ilustrações de talo em Hale (1976); detalhe do córtex na Figura 1a.

Talo verde cinzento claro em herbário, com até $8 \mathrm{~cm}$ diâm. Lobos de ramificação irregular a parcialmente dicotômica anisotômica, 1,5-4 mm larg., muito adnatos, aderidos, sobrepostos lateralmente a amontoados, de ápices arredondados, a margem crenada com linha negra sutil, as axilas ovais, a superfície contínua, lisa a subrugosa. Lacínulas ou lóbulos adventícios ausentes. Cílios ausentes. Máculas fracas, puntiformes, laminais. Pseudocifelas ausentes. Sorédios e pústulas ausentes. Isídios $0,1-0,5 \times$ ca. $0,05 \mathrm{~mm}$, em geral cilíndricos lisos, simples a parcialmente pouco ramificados, laminais (não abundantes), eretos, caducos, concoloridos, escurecidos ou de ápices amarronzados, sem ornamentações. Medula branca, sem manchas de pigmentos $\mathrm{K}+$ púrpura. Lado de baixo negro, lustroso, rugoso ou venado, pouco papilado, zona marginal marrom escura ou às vezes negra, atenuada, lustrosa, lisa a rugosa ou raramente venada, parcialmente papilada. Rizinas $0,1-0,6$ $\times$ ca. $0,05 \mathrm{~mm}$, negras, em geral simples ou algumas vezes irregularmente ramificadas, homogeneamente distribuídas, frequentes. Apotécios e picnídios não encontrados.
Substâncias de importância taxonômica: atranorina e cloroatranorina (córtex superior, $\mathrm{K}+$ amarelo, UV-), ácido protocetrárico e traços de substâncias não identificadas (medula, $\mathrm{K}+$ fraco amarelado, $\mathrm{C}-, \mathrm{KC}+$ róseo, $\mathrm{P}+$ alaranjado, UV-).

Material estudado: São Paulo, Serra da Cantareira, Parque Estadual da Cantareira, VI.2000, M.N. Benatti $1390 b$ (SP).

Ocorre na Ásia, África, América do Norte, América Central, e América do Sul. No Brasil, esta espécie foi citada para os estados de BA, MG, MS, MT, PA, PR, RJ e SP (Eliasaro 2001; Feuerer 2008; Fleig \& Riquelme 1991; Hale 1976; Kalb 1981; Lynge 1914; Jungbluth 2006; Marcelli 1998b, 2004; Ribeiro 1998; Swinscow \& Krog 1988; Vareschi 1973).

Esta espécie pode ser identificada pela formação de isídios laminais e pela presença de ácido protocetrárico na medula (é a única espécie do gênero encontrada na localidade com esta substância). Isídios menores escurecidos podem ser facilmente observados no único espécime encontrado. Pode ser diferenciada de Parmelinella cinerascens que apresenta curtos cílios axilares e tem como constituinte medular ácido salazínico ( $\mathrm{K}+$ amarelo $\Downarrow$ vermelho escuro, $\mathrm{P}+$ amarelado). Difere também de Canoparmelia caroliniana que tem ácido perlatólico medular ( $\mathrm{KC}+$ violáceo, $\mathrm{P}-)$, e córtex mais evidentemente maculado e bastante rachado.

Oúnico espécime de $C$. amazonica encontrado é um talo ramulícola, que devido ao pouco substrato, está com os lobos bastante sobrepostos 
ou amontoados. Quando encontrado sobre córtex arbóreo tem os lobos mais contíguos, como em formações de cerrados (Jungbluth 2006), onde pode também ser encontrada com mais frequência, apresentando inclusive talos férteis.

Canoparmelia caroliniana (Nyl.) Elix \& Hale, Mycotaxon 27: 278. 1986. = Parmelia caroliniana Nyl., Flora 68: 614. 1885. Tipo: ESTADOS UNIDOS DA AMÉRICA. CAROLINA DO SUL: leg. Ravenel 404 (H, lectótipo n.v.; FH-Tuck, duplicata n.v.). Ilustrações de talo em Hale (1976); detalhe do córtex na Figura $1 b$.

Talo verde cinzento (às vezes escuro) em herbário, com até $13 \mathrm{~cm}$ diâm. Lobos de ramificação dicotômica anisotômica a irregular, 1-4 mm larg., adnatos, aderidos, contíguos a ocasionalmente sobrepostos lateralmente, de ápices arredondados, a margem lisa a pouco sinuosa, crenada com linha negra sutil, as axilas ovais, a superfície contínua nas partes distais tornando-se rachada com quebras irregulares no centro, lisa a subrugosa. Lacínulas ou lóbulos adventícios ausentes. Cílios ausentes. Máculas distintas nas partes distais a fracas no centro, lineares irregulares a \pm reticulares, laminais a submarginais. Pseudocifelas ausentes. Sorédios e pústulas ausentes. Isídios $0,1-0,6 \times$ ca. 0,05 mm, em geral cilíndricos lisos, simples a ramificados ou raramente coralóides, laminais (moderados a abundantes), eretos, caducos, concoloridos, de ápices amarronzados ou raramente enegrecidos, sem ornamentações. Medula branca, sem manchas de pigmentos $\mathrm{K}+$ púrpura. Lado de baixo negro, às vezes com manchas marrons escuras, lustroso, rugoso ou venado, papilado, zona marginal marrom, atenuada, lustrosa, lisa a rugosa ou venada, parcialmente papilada. Rizinas 0,1-0,4 × ca. 0,05 mm, negras, às vezes marrons ou brancas quando próximas às margens, simples, homogeneamente distribuídas, frequentes. Apotécios (raros; encontrados somente em três espécimes) côncavos, sésseis, 0,3-3,5 mm diâm., laminais a submarginais, margem e anfitécio lisos tornando-se isidiados quando maduros, disco marrom claro, imperfurado; ascósporos elipsóides, 9-14 × (6-)7-8 $\mu \mathrm{m}$, epispório ca. $1 \mu \mathrm{m}$. Picnídios (encontrados somente em poucos espécimes) de ostíolo negro, imersos, submarginais a raramente laminais, conídios baciliformes a bifusiformes sutis 5-6 × ca. $1 \mu \mathrm{m}$.

Substâncias de importância taxonômica: atranorina e cloroatranorina (córtex superior,
$\mathrm{K}+$ amarelo, UV-), ácido divaricático e traços de substâncias não identificadas (medula, $\mathrm{K}-, \mathrm{C}-, \mathrm{KC}+$ violáceo, P-, UV+ branco azulado).

Material estudado: São Paulo, Serra da Cantareira, Parque Estadual da Cantareira, V.2000, M.N. Benatti 7, 12, 13, 1020 (SP); VI.2000, M.N. Benatti 1269, 1287, 1398 (SP); nas proximidades do Lago das Carpas, 9.I.1991, M.P. Marcelli et al. 10944, 10945, 10961, 10970, 10974, 10983, 10986, 10987 (SP); Bairro da Cachoeira, próximo da Rodovia Fernão Dias, 20.VII.1990, M.P. Marcelli et al. 9718, 9719, 9730, 9734, 9735, 9736, 9738, 9740 (SP); à beira da Estrada da Chapada, 09.I.1991, M.P. Marcelli et al. 11011 (SP); mata de Araucaria angustifolia no caminho para o Pé-deGalinha, 25.VI.1991, M.P. Marcelli et al. 11451 (SP); nas proximidades do lago perto da administração do parque, 15.VII.1991, M.P. Marcelli \& A. Rezende 11808 (SP); estrada principal para o lago das carpas, 30.III.1992, M.P. Marcelli et al. 13414 (SP). Mairiporã, Serra da Cantareira, construção de condomínio, 11.III.1989, M.P. Marcelli 6020, 6021, 6069 (SP).

Ocorre na Oceania, Europa, África, América do Norte, América Central e América do Sul. No Brasil, esta espécie foi citada para os estados de MG, MS, PR, RJ, RS, SC e SP (Brodo et al. 2001; Canêz 2005; Eliasaro 2001; Feuerer 2008; Fleig \& Riquelme 1991; Gumboski \& Eliasaro 2011; Hale 1976; Jungbluth 2006; Marcelli 2004; Osorio 1989; Pereira \& Marcelli 1989; Ribeiro 1998; Spielmann 2005, 2006; Swinscow \& Krog 1988) e por (Lynge 1924; Hale 1960) como o sinônimo Parmelia isidiophora Zahlbr., e Zahlbruckner 1926, como o sinônimo Parmelia luteola Zahlbr.

As principais características desta espécie são os isídios laminais simples a pouco ramificados, o córtex superior reticulado maculado (visível principalmente nas partes jovens distais) e normalmente bastante rachado, e a reação química $\mathrm{KC}+$ violácea na medula, pela presença de ácidos perlatólico como substância principal. É a espécie do gênero, bem como possivelmente de Parmeliaceae, mais facilmente encontrada na localidade de estudo. Tal como mencionado por Hale (1976a), esta é ainda a única espécie isidiada do gênero contendo ácido perlatólico medular.

Os espécimes M.N. Benatti 13, 1020, e M.P. Marcelli 10944, 10945 apresentam uma fraca reação $\mathrm{K}+$ amarelada na medula, que provavelmente se deve a pequenas concentrações de atranorina na porção superior desta, uma vez que não foram encontradas outras substâncias $\mathrm{K}+$ em cromatografia. Outro fato curioso sobre estes espécimes é que apesar de apresentarem ácido perlatólico medular, também visto em $\mathrm{CCD}$, 

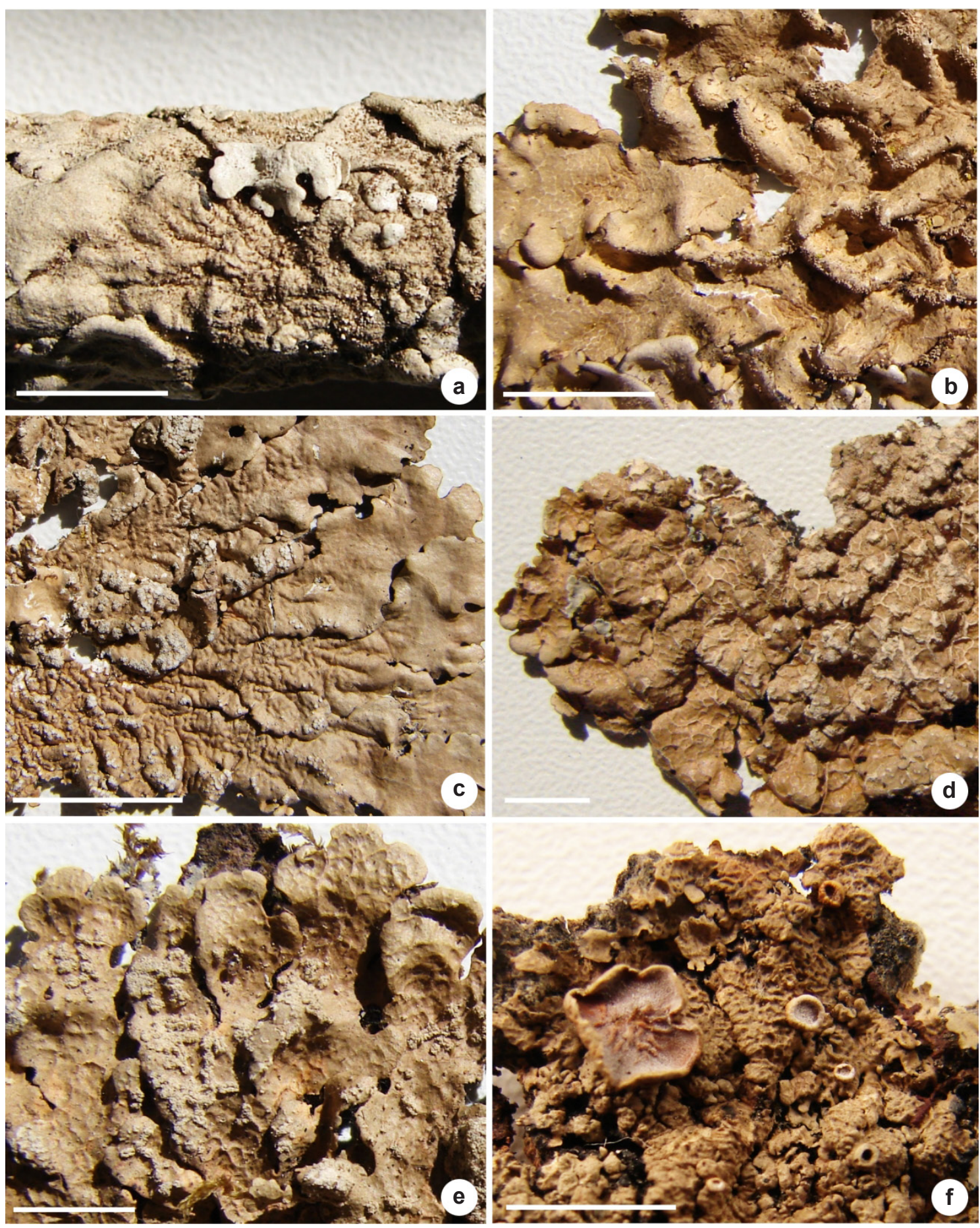

Figura 1 - Detalhes dos córtices superiores em espécimes de Canoparmelia - a. córtex superior de Canoparmelia amazonica (M.N. Benatti 1390b); b. córtex superior de Canoparmelia caroliniana (M.P. Marcelli 10961); c. córtex superior de Canoparmelia texana (M.N. Benatti 322); d. córtex superior de Crespoa carneopruinata (M.P. Marcelli 9744a); e. córtex superior de Crespoa crozalsiana (M.P. Marcelli 13536); f. córtex superior de Crespoa scrobicularis (M.P. Marcelli 16919). Barra $=5 \mathrm{~mm}$.

Figure 1 - Details of the upper cortices of Canoparmelia specimens - a. upper cortex of Canoparmelia amazonica (M.N. Benatti 1390b); b. upper cortex of Canoparmelia caroliniana (M.P. Marcelli 10961); c. upper cortex of Canoparmelia texana (M.N. Benatti 322); d. upper cortex of Crespoa carneopruinata (M.P. Marcelli 9744a); e. upper cortex of Crespoa crozalsiana (M.P. Marcelli 13536); f. upper cortex of Crespoa scrobicularis (M.P. Marcelli 16919). Bars $=5 \mathrm{~mm}$. 
é difícil visualizar a reação $\mathrm{KC}+$ rósea, mais efêmera do que nos demais espécimes estudados (ver comentários em Jungbluth 2006). A reação $\mathrm{UV}+$ é às vezes difícil de constatar, devido a medula ser variavelmente reflexiva. Alguns espécimes apresentam uma maculação forte sobre trechos do córtex que ficam venados, tal como facilmente visto nos espécimes M.P. Marcelli 9730, 9734 e 10987.

Dentre as espécies mais semelhantes encontradas na localidade de estudo, Canoparmelia amazonica (Nyl.) Elix \& Hale difere pelo córtex superior mais contínuo e emaculado/fraco maculado, e por apresentar ácido protocetrárico medular $(\mathrm{K}+$ fraco amarelo, $\mathrm{KC}$ ou $\mathrm{KC}+$ rosa a alaranjado evanescente, $\mathrm{P}+$ laranja). Parmelinella cinerascens (Lynge) Benatti \& Marcelli difere pela ausência ou escassez de máculas, presença de cílios curtos axilares e formação de ácidos salazínico e consalazínico medulares $(\mathrm{K}+$ amarelo $\$ vermelho, $\mathrm{P}+$ alaranjado).

Canoparmelia sanguinea Marcelli, Benatti \& Elix. Mycotaxon 106: 436. 2008. Tipo: BRASIL. SÃO PAULO: Mairiporã, Cantareira Range, village construction, 03.XI.1989, leg. M.P. Marcelli 6029 (SP!, holótipo). Ilustrações em Benatti et al. (2008).

Talo verde acinzentado escuro a pardo em herbário, sublobado, com até $9 \mathrm{~cm}$ diâm. Lobos de ramificação subdicotômica a irregular, 1-3,5 $\mathrm{mm}$ larg., muito adnatos, aderidos, contíguos a sobrepostos lateralmente a \pm amontoados no centro, de ápices subarredondados a parcialmente subtruncados, a margem parcialmente subcrenada, as axilas ovais, a superfície contínua a irregularmente quebrada, lisa nas partes distais tornando-se frequentemente rugosa ou sutilmente escrobiculada especialmente em partes velhas. Lacínulas ou lóbulos adventícios ausentes ou em partes velhas, simples, curtos, subtruncados, planos. Cílios ausentes. Máculaslaminais, mais distintas nas partes distais, lineares irregulares, confluentes, por vezes originando quebras. Pseudocifelas ausentes. Pústulas e sorédios ausentes. Isídios $0,05-0,50 \times \mathrm{ca}$. $0,05-0,10 \mathrm{~mm}$, granulares a cilíndricos lisos e curtos, simples a parcialmente ramificados, em geral muito aglomerados (não coralóides), laminais, eretos, firmes, concoloridos, parcialmente escurecidos ou de ápices amarronzados, eciliados. Medula branca, sem manchas de pigmentos $\mathrm{K}+$ púrpura. Lado de baixo mesclado, negro a marrom escuro, lustroso a opaco, de liso a rugoso ou ocasionalmente pouco venado, papilado, zona marginal marrom, atenuada, lustrosa, lisa a rugosa ou venada, nua a esparsamente papilada tornando-se rizinada em direção ao centro. Rizinas $(0,1-)$ 0,2-0,7(-1) × ca. $0,05 \mathrm{~mm}$, marrons escuras a negras, marrons ou brancas junto às margens, simples a raramente furcadas, frequentes, homogeneamente distribuídas. Apotécios não encontrados. Picnídios raros, submarginais, de ostíolos negros, conídios não encontrados.

Substâncias de importância taxonômica: atranorina (córtex superior, $\mathrm{K}+$ amarelo, UV-), complexo formado por diversos ácidos medulares (Benatti et al. 2008), sendo o principal o ácido olivetolcarboxílico (medula, K-, C+ vermelho, $\mathrm{KC}+$ vermelho, P-, UV-).

Material adicional estudado: Mairiporã, Serra da Cantareira, construção de condomínio, 3.XI.1989, M.P. Marcelli 6028 (B!, parátipo, SP!, isoparátipo).

Ocorre na América do Sul. No Brasil é citada para os estados do RS e SP (Benatti et al. 2008, Canêz et al. 2009).

Esta espécie foi descrita há poucos anos (Benatti et al. 2008) a partir de dois exemplares encontrados na Serra da Cantareira, e um ano depois foi encontrada no estado do Rio Grande do Sul por Canêz et al. (2009). É morfologicamente muito semelhante à Canoparmelia caroliniana, porém difere pela coloração mais escura do talo, perceptível tanto no material visto em campo como herborizado, e notavelmente pela composição química medular, muito mais complexa, contendo diversos ácidos os quais não incluem o ácido divaricático (notável pelos testes de coloração $\mathrm{C}$ e KC que reagem fortemente avermelhados). Para mais comentários e comparações, ver a descrição original em Benatti et al. (2008).

Canoparmelia texana (Tuck.) Elix \& Hale. Mycotaxon 27: 279. 1986. $\equiv$ Parmelia texana Tuck., American Journal of Science and Arts, series 2, 25: 424. 1858. Tipo: ESTADOS UNIDOS DA AMÉRICA. TEXAS: thickets of the Blanco, s.d., Wright (FH, lectótipo n.v., M, US, duplicatas n.v.). Ilustrações de talo em Hale (1976); detalhe do córtex na Figura 1c.

Talo cinza esverdeado claro em herbário, lobado a sublaciniado, com até $11 \mathrm{~cm}$ diâm. Lobos de ramificação dicotômica anisotômica a irregular, 1-4(-5) mm larg., adnatos, pouco aderidos, contíguos a sobrepostos lateralmente ou raramente amontoados, de ápices arredondados a subtruncados, a margem \pm crenada com linha 
negra sutil, as axilas ovais, a superfície contínua e lisa nas partes distais tornando-se variavelmente rugosa e irregularmente quebrada no centro. Lacínulas ou lóbulos adventícios ausentes. Cílios ausentes. Máculas ausentes no centro, fracas ou distintas nas partes distais, lineares irregulares, por vezes originando quebras. Pseudocifelas ausentes. Pústulas e isídios ausentes. Sorais orbiculares a capitados ou subirregulares, laminais a submarginais não densos, claros, sorédios granulares, aglutinados, às vezes tornando-se corticados. Medula branca, sem manchas de pigmentos $\mathrm{K}+$ púrpura. Lado de baixo negro, lustroso, de liso a rugoso ou ocasionalmente venado, papilado, zona marginal marrom, atenuada, lustrosa, lisa a venada, papilada. Rizinas $0,1-1 \times$ ca. $0,05 \mathrm{~mm}$, negras a marrons escuras ou às vezes brancas junto às margens, simples a raramente furcadas, poucas a frequentes, homogeneamente distribuídas. Apotécios (raros; encontrados somente em um espécime) côncavos, sésseis, 0,2-1,9 mm diâm., laminais a submarginais, margem e anfitécio lisos tornando-se sorediados quando maduros, disco marrom claro, imperfurado; ascósporos elipsóides, 9-11,5 × (5-)6-7 $\mu \mathrm{m}$, epispório ca. 1 $\mu \mathrm{m}$. Picnídios não encontrados.

Substâncias de importância taxonômica: atranorina (córtex superior, $\mathrm{K}+$ amarelo, $\mathrm{UV}-$ ), ácido divaricático (medula, K-, C-, KC- ou + fraco violáceo, P-, UV+ azulado claro).

Material estudado: São Paulo, Serra da Cantareira, Parque Estadual da Cantareira, na mata, V.2000, M.N. Benatti 322 (SP); sobre a pedra grande ao lado do museu, VI.2000, M.N. Benatti 1373, 1382 (SP); sobre a Pedra Grande, 9.I.1991, M.P. Marcelli et al. 10916 (SP); mata de Araucaria angustifolia no caminho para o Pé-deGalinha, 25.VI.1991, M.P. Marcelli et al. 11453 (SP); próximo ao lago perto da administração, 16.VII.1991, M.P. Marcelli, A. Rezende, O. Yano \& F.M.M. Coppolla 11763, 11783 (SP); estrada principal para o lago das carpas, 30.III.1992, M.P. Marcelli et al. 13400 (SP); Bairro da Cachoeira, próximo da Rodovia Fernão Dias, 20.VII.1990, M.P. Marcelli et al. 9724, 9725, 9728, 9737, 9739, 9774 (SP). Mairiporã, Serra da Cantareira, construção de condomínio, 11.III.1989, M.P. Marcelli 6053 (SP).

Ocorre na Oceania, Ásia, África, América do Norte, América Central, e América do Sul. No Brasil, a espécie é citada para os estados de MG, MS, PR, RS, SC e SP (Brodo et al. 2001; Calvelo \& Liberatore 2002; Canêz 2005; Divakar \& Upreti 2005; Eliasaro 2001; Elix 1994; Esslinger \& Egan 1995; Fleig \& Riquelme 1991; Gumboski
\& Eliasaro 2011; Hale 1976; Jungbluth 2006; Marcelli 1991, 2004; Osorio 1972, 1989; Ribeiro 1998; Spielmann 2005, 2006; Swinscow \& Krog 1988; Vareschi 1973).

É uma das espécies mais facilmente encontrada em áreas urbanas não litorâneas do Brasil, tendo sido estudada quanto ao seu alto grau de tolerância a poluentes para uso como bioindicador de poluição aérea (e.g., Saiki et al. 1997; Coccaro et al. 2000). Esta espécie pode ser identificada pelos sorais laminais capitados, talo de centro rugoso e quebrado, e pela presença de ácido divaricático na medula. A reação normalmente é difícil de ser percebida, devido a medula ser muito fina e facilmente encharcar com os reagentes, mas pode ser notada se os reagentes forem colocados com cuidado, apenas de leve. Apenas um único espécime fértil foi encontrado, o maior talo, todavia contendo vários apotécios.

Há vários talos com características um tanto variáveis: alguns são mais lobados, tem córtex inferior mais venado e rizinas brancas com mais frequência, enquanto outros podem ser mais sublaciniados, tem córtex inferior mais liso ou não apresentam rizinas brancas. Foram encontrados alguns poucos espécimes saxícolas, que apresentam sorédios granulares parcialmente corticados. Nestes espécimes observou-se com mais facilidade que a presença de rizinas brancas e as partes venadas dispostas concentricamente nas margens do córtex inferior, mais acentuadas que vistas na maioria dos espécimes corticícolas. Não há outras diferenças significativas para com os demais espécimes.

Alguns espécimes podem apresentar sorais mais aglomerados e parcialmente enegrecidos, decorrentes de sorédios que não se desprenderam e que ficaram em seus sorais. Somente um único espécime fértil foi encontrado, as características de apotécios e ascósporos estão de acordo com as descritas por Hale (1976a). Esta foi a única espécie sorediada do gênero encontrada na área de estudo.

Crespoa carneopruinata (Zahlbr.) Lendemer \& Hodkinson, North American Fungi 7: 3. 2012. Parmelia carneopruinata Zahlbr., Sitzungs. Kaiser. Akad. Wissen. Math. Natur. Klasse 1: 419. 1902. Tipo: BRASIL. RIO DE JANEIRO, leg. Höhnel 164 (lectótipo W, n.v.). Ilustrações de talo em Hale (1976); detalhe das estruturas estão na Figura 1d.

Talo cinza esverdeado a cinza pardo em herbário, laciniado a sublaciniado, com até 14 $\mathrm{cm}$ diâm. Lacínias de ramificação dicotômica 
anisotômica a irregular, $(0,5-) 1-2(-2,5) \mathrm{mm}$ larg., adnatos, adpressos, contíguas a raramente sobrepostas lateralmente, de ápices subtruncados a raramente subarredondados, a margem crenada ou irregular sem linha negra, as axilas ovais a irregulares, a superfície foveolada, continua nas partes distais tornando-se quebrada em direção ao centro, as quebras originadas nos fovéolos. Lacínulas ou lóbulos adventícios ausentes. Máculas normalmente distintas, laminais, reticulares a irregulares, principalmente sobre os fovéolos, originando quebras. Pseudocifelas ausentes. Cílios ausentes. Pústulas e Isídios ausentes. Sorais orbiculares a capitados ou subirregulares, adensando sobre as quebras dos fovéolos, laminais a submarginais densos, claros, sorédios subgranulares. Medula branca. Lado de baixo negro, lustroso, liso a rugoso, zona marginal marrom e atenuada a ocasionalmente negra e indistinta do centro, lustrosa, lisa a rugosa, papilada a parcialmente rizinada. Rizinas $0,1-0,8$ $\times$ ca. $0,05 \mathrm{~mm}$, negras, simples a raramente furcadas ou \pm esquarosas, frequentes a abundantes, homogeneamente distribuídas. Apotécios não encontrados. Picnídios raros, laminais, de ostíolos marrons a negros, conídios não encontrados.

Substâncias de importância taxonômica: atranorina (córtex superior, $\mathrm{K}+$ amarelo, UV-), ácidos estíctico, conestíctico, outras substâncias do grupo químico do ácido estíctico (vide Jungbluth 2006), e traços variáveis de ácido menegaziaico (medula, K+amarelo, C-, KC-, P+ salmão, UV-).

Material estudado: São Paulo, Serra da Cantareira, Sítio da Cachoeira, próximo a Rodovia Fernão Dias, 16.VII.1991, leg. M.P. Marcelli et al 9744a (SP); Parque Estadual da Cantareira, à beira da Estrada da Chapada, 09.I.1991, leg. M.P. Marcelli et al. 1001 (SP); Parque Estadual da Cantareira, próximo ao lago perto da administração, 16.VII.1991, leg. M.P. Marcelli et al. 11753 (SP); mata de encosta, 9.XII.1991, leg. M.P. Marcelli et al. 12526, 12527 (SP).

Ocorre na Ásia, Europa, América do Norte, América Central e América do Sul. No Brasil, esta espécie foi citada para os estados de MG, RJ, RS, SC e SP (Awasthi 1976; Canêz 2005; Divakar \& Upreti 2005; Gumboski \& Eliasaro 2011; Hale 1976; Jungbluth 2006; Nash \& Elix 2002; Osorio 1992, 1998; Ribeiro 1998; Spielmann 2006; Zahlbruckner 1902).

Crespoa carneopruinata é caracterizada pelo talo fortemente foveolado, sorais laminais orbiculares e presença de ácidos do complexo stictico na medula. Esta espécie é bastante similar à C. crozalsiana, também encontrada na área de estudo, e tal como mencionado em Hale (1976a) e Jungbluth (2006), estas espécies são as vezes de difícil distinção, uma vez que compartilham diversas características.

Crespoa carneopruinata apresenta lacínias muito estreitas de ápices truncados, e um hábito constante dos sorais de se desenvolverem seguindo o padrão de quebras originado pelas máculas sobre os fovéolos. Em C. crozalsiana, os lobos são mais largos e arredondados (em média o dobro da largura ou mais, ver descrição), além de que os sorais tendem a somente coalescer quando em maior número, formando um aglomerado ao invés de acompanharem as quebras.

Segundo Jungbluth (2006) esta espécie pode apresentar cílios axilares esparsos, que não ultrapassam meio milímetro de comprimento. Estas estruturas não foram visualizadas nos espécimes estudados, e seu desenvolvimento pode ser aleatório.

Crespoa crozalsiana (B. de Lesd. ex Harm.) Lendemer \& Hodkinson, North American Fungi 7: 3. 1986. $\equiv$ Parmelia crozalsiana de Lesd., Lichens de France 4: 555. 1910. Tipo: FRANÇA. HÉRAULT: Agde, V.1909, De Crozals (lectótipo US, n.v.). Ilustrações de talo em Hale (1976); detalhe do córtex na Figura 1e.

Talo cinza esverdeado a cinza pardo em herbário, sublobado a lobado, com até 11 $\mathrm{cm}$ diâm. Lobos de ramificação dicotômica anisotômica a irregular, (1,5-)2-5 mm larg., adnatos, adpressos, contíguas a ocasionalmente sobrepostas lateralmente, de ápices arredondados a subarredondados, a margem crenada sem linha negra, as axilas ovais, a superfície foveolada, continua nas partes distais tornando-se quebrada em direção ao centro, as quebras originadas nos fovéolos. Lacínulas ou lóbulos adventícios ausentes. Máculas normalmente distintas, laminais, reticulares a irregulares, principalmente sobre os fovéolos, originando quebras. Pseudocifelas ausentes. Cílios ausentes. Pústulas e Isídios ausentes. Sorais orbiculares a capitados, às vezes aglomerando-se sobre o córtex tornando-se irregulares, laminais, claros, sorédios farinhosos a subgranulares. Medula branca. Lado de baixo negro, lustroso, liso a rugoso ou venado, zona marginal marrom clara, atenuada, lustrosa, lisa a rugosa, papilada a parcialmente rizinada. Rizinas $0,1-0,7$ $\times$ ca. $0,05 \mathrm{~mm}$, negras a ocasionalmente brancas, 
simples a raramente furcadas ou \pm esquarosas, frequentes a abundantes, homogeneamente distribuídas. Apotécios e Picnídios não encontrados.

Substâncias de importância taxonômica: atranorina (córtex superior, $\mathrm{K}+$ amarelo, UV-), ácidos estíctico, conestíctico, outras substâncias do grupo químico do ácido estíctico (vide Jungbluth 2006), e traços variáveis de ácido menegaziaico (medula, K+amarelo, C-, KC-, P+ salmão, UV-). Material estudado: São Paulo, Parque Estadual da Cantareira, Bairro Cachoeira, próximo à Rodovia Fernão Dias, 20.VII.1990, leg. M.P. Marcelli et al. 9753 (SP); próximo ao Lago das Carpas, 25.VI.1991, leg. M.P. Marcelli et al. 11425, 11426, 11427, 11575 (SP); Núcleo da Pedra Grande, 18.V.1992, leg. M.P. Marcelli et al. 13536 (SP); Parque Estadual da Cantareira, VI.2000, leg. M.N. Benatti 1004 (SP).

Ocorre na Oceania, Ásia, Europa, África, América do Norte e América do Sul. No Brasil, esta espécie foi citada para os estados de MG, MS, PR, RS e SP (Calvelo \& Liberatore 2002; Canêz 2005; Eliasaro 2001; Elix 1994; Esslinger \& Egan 1995; Hale 1976; Jungbluth 2006; Marcelli 1991, 2004; Osorio 1970b, 1973, 1975; Pereira \& Marcelli 1989; Ribeiro 1998; Spielmann 2005, 2006; Swinscow \& Krog 1988; Vareschi 1973).

Esta espécie é caracterizada pelos lobos de ápices arredondados, córtex superior foveolado e maculado com quebras frequentes, sorais orbiculares laminais e presença de ácidos do complexo estictico na medula. Para mais comparações ver comentários em Crespoa carneopruinata.

Os espécimes M.P. Marcelli 11425, 11426 , 11427,11575 são saxícolas, mas não apresentam diferenças significativas para com os demais espécimes corticícolas estudados.

Crespoa scrobicularis (Kremp.) Benatti \& Lendemer, Brittonia: 66: 287-291. 三 Parmelia scrobicularis Kremp., Videnskabelige Meddelelser Naturhistorik Forening Kjöbenhavn 25: 10. 1873. Tipo: BRASIL. MINAS GERAIS, Lagoa Santa, Warming s.n. (M!, lectótipo). Ilustrações de talo em Hale (1976); detalhe do córtex na Figura 1f.

Talo cinza esverdeado pardo em herbário, com até $4 \mathrm{~cm}$ diâm. Lacínias de ramificação dicotômica anisotômica a irregular, 0,5-1,5 mm larg., adnatas e adpressas, contíguas a ocasionalmente sobrepostos lateralmente, de ápices truncados a subtruncados, a margem sinuosa, crenada, sem linha negra, as axilas ovais a irregulares, a superfície contínua, com raras quebras ocasionais em partes velhas, escrobiculada. Lóbulos ou lacínulas adventícias ausentes. Máculas distintas, lineares a reticulares, laminais, acompanhando o relevo saliente do córtex. Pseudocifelas ausentes. Cílios ausentes. Sorédios, pústulas e isídios ausentes. Medula branca, sem manchas de pigmentos $\mathrm{K}+$ púrpura. Lado de baixo negro, opaco a pouco lustroso, subrugoso, zona marginal marrom a negra, atenuada a indistinta do centro, pouco lustrosa, lisa a subrugosa, papilada, tornando-se rizinada em direção ao centro. Rizinas $0,1-0,6 \times$ ca. $0,05 \mathrm{~mm}$, negras, simples ou raramente com ramificações irregulares, abundantes, agrupadas. Apotécios planos a subcôncavos, 0,5-4 mm diâm., sésseis a adnatos, laminais a submarginais, margem lisa, anfitécio liso tornando-se rugoso, não ornamentados, disco marrom claro, imperfurado, normalmente pruinoso, ascósporos elipsoides a ovais, $10-14 \times 8-10 \mu \mathrm{m}$, epispório ca. $1 \mu \mathrm{m}$. Picnídios laminais a submarginais, negros, imersos, de ostíolo negro. Conídios filiformes, (12-)14-23 $\times$ ca. $0,5 \mu \mathrm{m}$.

Substâncias de importância taxonômica: atranorina (córtex superior, $\mathrm{K}+$ amarelo, UV-), ácidos estíctico, conorestíctico e menegaziaico (medula, K + amarelo, C-, KC-, P+ salmão, UV-). Material estudado: Mairiporã, Serra da Cantareira, mata de encosta, II.1981, M.P. Marcelli 16919 (SP).

Ocorre na Oceania e América do Sul. No Brasil, esta espécie foi citada para os estados de MG, MS, PA, PR e SP (Calvelo \& Liberatore 2002; Eliasaro 2001; Hale 1976; Feuerer 2008; Osório 1973; Ribeiro 1998).

Somente um espécime foi encontrado na área de estudo. As principais características são: o talo de lacínias estreitas, a ausência de propágulos vegetativos, o córtex superior fortemente escrobiculado, os apotécios de discos marrons claros normalmente recobertos por pruína branca, e a presença de ácidos do complexo estíctico na medula. Mesmo apotécios muito jovens apresentam normalmente o disco recoberto pela pruína.

O material apresenta muitos apotécios e picnídios, contendo ascósporos e conídios maduros e que estão de acordo com os mencionados por Jungbluth (2006) para espécimes de áreas de cerrado. Esta foi a única espécie deste grupo de Parmeliaceae encontrada na localidade a apresentar reprodução somente pela formação de apotécios, todas as demais sendo sorediadas ou isidiadas. É aparentemente relacionada pela morfologia (principalmente o aspecto do córtex superior) e pela química medular (ácidos do complexo estíctico) à Crespoa carneopruinata e à C. crozalsiana . 


\section{Agradecimentos}

$\mathrm{O}$ autor agradece à assessoria, a revisão do artigo e à FAPESP (Fundação para o Amparo à Pesquisa do Estado de São Paulo), a concessão da Bolsa de Iniciação Científica nº 00/01009-1.

\section{Referências}

Asahina, Y. \& Shibata, S. 1954. Chemistry of Lichen substances. Japan Society for the Promotion of Science, Tóquio. 240p.

Awasthi, D.D. 1976. Lichen genus Parmelia in India ISubgenera Parmelia and Amphigymnia. Biological Memoirs 1: 155-229.

Benatti, M.N. 2012. Pequenas espécies de Parmeliaceae ciliadas no Parque Estadual da Cantareira, Estado de São Paulo, Brasil: gêneros Bulbothrix, Parmelinella e Parmelinopsis (Parmeliaceae, Ascomycota). Hoehnea 39: 207-218.

Benatti, M.N. \& Jungbluth, P. 2014. Physciaceae foliosas do Parque Estadual da Cantareira, estado de São Paulo, Brasil. I. Gêneros Dirinaria, Hyperphyscia e Pyxine. Iheringia 69: 17-27.

Benatti, M.N. \& Lendemer, J.C. 2014. Canoparmelia scrobicularis belongs to the genus Crespoa (Parmeliaceae, lichenized Ascomycota). Brittonia 66: 287-291.

Benatti, M.N. \& Marcelli, M.P. 2007. Gêneros de fungos liquenizados dos manguezais do Sul-Sudeste do Brasil, com enfoque no manguezal do Rio Itanhaém, Estado de São Paulo. Acta Botanica Brasilica 21: 863-878.

Benatti, M.N. Marcelli, M.P. \& Elix, J.A. 2008. Canoparmelia sanguinea, a new Parmeliaceae from Brazil. Mycotaxon 106: 435-439.

Brodo, I.M., Sharnoff, S.D. \& Sharnoff, S. 2001. Lichens of North America. Yale University Press, New Haven. 795p.

Bungartz, F. 2001. Analysis of lichen substances. Disponível em $<$ http://ces.asu.edu/ASULichens/plb 400/laboratory/ chemistry/tlc.html>. Acesso em 1 outubro 2004.

Calvelo, S. \& Liberatore, S. 2002. Catálogo de los Líquenes de la Argentina. Kurtziana 29: 7-170.

Canêz, L.S. 2005. A família Parmeliaceae na localidade de Fazenda da Estrela, município de Vacaria, Rio Grande do Sul, Brasil. Dissertação de Mestrado. Instituto de Botânica, São Paulo. 274p.

Canêz, L.S.; Marcelli, M.P. \& Elix, J.A. 2009. New Brazilian species of Canoparmelia with medullary olivetoric, anziaic, and sekikaic complexes. Mycotaxon 110: 465-472.

Clauset, L.R. \& Soares, D. 1999. Paisagem Paulista: Áreas Protegidas. Empresa das Artes, São Paulo. 185p.

Divakar, P.K. \& Upreti, D.K. 2005. Parmelioid Lichens in India (a revisionary study). Bishen Singh Mahendra Pal Singh, Dehra Dun. 448p.
Eliasaro, S. 2001. Estudio taxonómico y florístico sobre las Parmeliaceae sensu stricto (Ascomycota Liquenizados) del segundo planalto del estado de Paraná, Brasil. Tese de Doutorado. Universidad de Buenos Aires, Buenos Aires. 269p.

Elix, J.A.; Johnston, J. \& Verdon, D. 1986. Canoparmelia, Paraparmelia and Relicinopsis, three new genera in the Parmeliaceae (lichenized Ascomycotina). Mycotaxon 27: 271-282.

Elix, J.A. 1994. Canoparmelia. In: Orchard, A.E. \& Grgurinovic, C. (eds.). Flora of Australia, Lichens. Introduction, Lecanorales 2. Vol. 55. Australia Government Publishing Service, Canberra. Pp. 21-31.

Esslinger, T.L. \& Egan, R.S. 1995. A sixth checklist of the lichen-forming, lichenicolous, and allied fungi of the continental states and Canada. The Bryologist 98: 467-549.

Feuerer, T.E. (ed.). 2008. Checklists of lichens and lichenicolous fungi. Versão 1 Setembro de 2008. Disponível em $<$ http://www.checklists.de $>$. Acesso em outubro 2008.

Fink, B. 1905. How to collect and study lichens. The Bryologist 8: 22-27.

Fleig, M. \& Riquelme, I. 1991. Liquens de Piraputanga, Mata Grosso do Sul, Brasil. Acta Botanica Brasilica 5: 3-12.

Galloway, D.J. 1985. Flora of New Zealand - lichens. Government Printer, Wellington. 662p.

Galloway, D.J. 2007. Flora of New Zealand Lichens. Revised second edition including lichen-forming and lichenicolous fungi. Vols. 1 and 2. Manaaki Whenua Press, Lincoln. 2261p.

Gumboski, E.L. \& Eliasaro, S. 2011. Checklist of lichenized fungi of Santa Catarina State (Brazil). Mycotaxon 115: 535.

Hale, M.E. 1960. A revision of the South American species of Parmelia determined by Lynge. Contributions from the United States National Herbarium 36: 1-41.

Hale, M.E. 1976. A monograph of the Lichen genus Pseudoparmelia Lynge (Parmeliaceae). Smithsonian Contributions to Botany 31: 1-62.

Hale, M.E. 1979. How to know the Lichens. The Pictured-Key Nature Series. W.C.Brown Company Publishers, Dubuque. 246p.

Hale, M.E. 1987. How to know the Lichens. $2^{\text {nd }} \mathrm{ed}$. WCB/ McGraw-Hill, Boston. 246p.

Huneck, S. \& Yoshimura, I. 1996. Identification of lichen substances. Springer, Berlin. 493p.

Jungbluth, P. 2006. A família Parmeliaceae (fungos liquenizados) em fragmentos de cerrados do estado de São Paulo. Dissertação de Mestrado. Instituto de Botânica, São Paulo. 323p.

Kalb, K. 1981. Lichenes Neotropici ausgegeben von Klaus Kalb. Fascikel I (No. 1-40). Neumarkt, 18 September 1981. 12p.

Lynge, B. 1914. Die Flechten der ersten Regnellschen Expedition. Die Gattungen Pseudoparmelia gen. 
nov. und Parmelia Ach. Arkiv för Botanik 13: 1-172.

Lynge, B. 1924. On some South American lichens of the genera Parmelia, Candelaria, Theloschistes and Pyxine. Nytt Magazin for Naturvidenskaberne 62: 83-97.

Malcolm, W.M. \& Galloway, D.J. 1997. New Zeland Lichens: checklist, key and glossary. Museum of New Zeland Te Papa Tongarewa. 192p.

Marcelli, M.P. 1991. Aspects of the foliose lichen flora of the southern-central coast of São Paulo State, Brazil. In: Galloway, D.J. (ed.). Tropical lichens: their systematics, conservation, and ecology, Systematics Association Special. Vol. 43. Clarendon Press, Oxford. Pp. 151-170.

Marcelli, M.P. 1998a. History and current knowledge of Brazilian Lichenology. In Marcelli, M.P. \& Seaward, M.R.D. (eds). Lichenology in Latin America: history, current knowledge and applications. CETESB, São Paulo. Pp. 25-45.

Marcelli, M.P. 1998b. Diversidade dos fungos liquenizados no estado de São Paulo: um diagnóstico. In: Joly, C.A. \& Bicudo, C.E.M. (eds.). Biodiversidade do estado de São Paulo, Brasil: síntese do conhecimento ao final do século XX. Vol. 2. FAPESP, São Paulo. Pp. 25-35.

Marcelli, M.P. 2004. Checklist of lichens and lichenicolous fungi of Brazil. Version 1: June 2004. Disponível em $<$ http://www.biologie.uni-hamburg.de/checklists/ brazil 1.htm>. Acesso em outubro 2004.

Nash III, T.H. \& Elix, J.A. 2002. Canoparmelia. In: Nash III, T.H.; Ryan, B.D.; Gries, C. \& Bungartz, F. (eds.). Lichen flora of the greater Sonoran desert Region. Vol. 1. Arizona State University, Tempe. Pp. 122-125.

Orange, A.; James, P.W. \& White, F.J. 2001. Microchemical methods for the identification of lichens. The British Lichen Society, London. 101p.

Osorio, H.S. 1970. Contribution to the lichen flora of Uruguay IV. Some lichens from northern Uruguay. Nova Hedwigia 19: 339-344.

Osorio, H.S. 1972. Contribution to the lichen flora of Uruguay. VII. A preliminary catalogue. Comunicaciones Botánicas del Museo de Historia Natural de Montevideo 4: 1-46.

Osorio, H.S. 1973. Contribution to the lichen flora of Brazil I. New or additional records. Revista da Faculdade de Ciências (Lisboa), $2^{\mathrm{a}}$ série C, Ciências Naturais 17: 447-450.

Osorio, H.S. 1975. Contribution to the Lichen flora of Uruguay. VIII. Additions and corrections. Comunicaciones Botánicas del Museo de Historia Natural de Montevideo 4: 1-12.
Osorio, H.S. 1989. Contribution to the lichen flora of Brazil. XXIII. Lichens from São Paulo city. Mycotaxon 36: 161-162.

Osorio, H.S. 1992. Contribucion a la flora liquénica del Uruguay. XXV. Líquens publicados entre 1972 a 1991. Anales del Museo Nacional de Historia Natural de Montevideo (Series 2) 8: 43-70.

Osorio, H.S. 1998. Contribución a la flora liquénica del Uruguay. XXX. Líquenes del Este de Uruguay. Comunicaciones Botánicas del Museo de Historia Natural de Montevideo 6: 1-12.

Pereira, W.R. \& Marcelli, M.P. 1989. Liquens da Reserva Biológica do Alto da Serra de Paranapiacaba. Acta Botanica Brasilica 3: 89-94.

Ribeiro, C.H. 1998. A família Parmeliaceae (Ascomycota liquenizados) em regiões montanhosas dos estados de Minas Gerais, Rio de Janeiro e São Paulo. Dissertação de Mestrado. Universidade de São Paulo, São Paulo. 194p.

São Paulo (Estado) 1988. Vegetação significativa do Município de São Paulo. Secretaria do Meio Ambiente (SMA) Secretaria Municipal de Planejamento (SEMPLA). São Paulo. 98p.

Spielmann, A.A. 2005. A família Parmeliaceae (fungos liquenizados) nos barrancos e peraus da encosta da Serra Geral, no Vale do Rio Pardo, Rio Grande do Sul, Brasil. Dissertação de Mestrado. Instituto de Botânica, São Paulo. 204p.

Spielmann, A.A. 2006. Checklist of lichens and lichenicolous fungi of Rio Grande do Sul (Brazil). Caderno de Pesquisa, Série Biologia (UNISC) 18: 7-125.

Swisncow, T.D.V. \& Krog, H. 1988. Macrolichens of East Africa. British Museum of Natural History, London. 390p.

Vareschi, V. 1973. Resultados liquenológicos de excursiones efectuadas en Venezuela ${ }^{\circ} 3$. Catálogo de los Líquenes de Venezuela. Acta Botanica Venezuelica 8: 177-245.

Walker, J.W. \& James, P.W. 1980. A revised guide to microchemical techniques for the identification of lichen products. British Lichen Society Bulletin 46 (supplement): 13-29.

White, F.J. \& James, P.W. 1985. A new guide to microchemical techniques for the identification of lichen substances. British Lichen Society Bulletin 57 (supplement): 1-41.

Zahlbruckner, A. 1902. Studien über brasilianische Flechten. Sitzungsberichte der MathematschNaturwissenschaftlichen Klasse der Kaiserlichen Academie der Wissenschaften. Abteilung I: 357-432.

Zahlbruckner, A. 1926. Catalogus Lichenum Universalis. Borntraeger, Leipzig. Pp. 1-480. 